\title{
LANGLANDS RECIPROCITY FOR ALGEBRAIC SURFACES
}

\author{
Victor Ginzburg, Mikhail Kapranov, and Eric Vasserot
}

Twenty-five years ago R. Langlands proposed [L] a "fantastic generalization" of Artin-Hasse reciprocity law in the classical class field theory. He conjectured the existence of a correspondence between automorphic irreducible infinite-dimensional representations of a reductive group $G$ over a global number field on the one hand, and (roughly speaking) finite dimensional representations of the Galois group of the field, on the other hand. Following an earlier idea of A. Weil, Langlands' conjecture was reinterpreted by V. Drinfeld [Dr 3] and G. Laumon [La] in purely geometric terms. In the complex geometry setup the number field gets replaced by a Riemann surface $X$, the Galois group of the field gets replaced by the fundamental group, $\pi_{1}(X)$, and an automorphic representation gets replaced by a perverse sheaf, cf. [BBD], on the moduli space of algebraic principal $G$-bundles on $X$.

The purpose of this note is to formulate some results and conjectures related to Langlands reciprocity for algebraic surfaces, as opposed to algebraic curves. That would amount, in number theory, to a non-abelian higher dimensional generalization of the class field theory, the subject that remains a total mystery at the moment. A key step of our geometric approach is a construction of Hecke operators for vector bundles on an algebraic surface. The main point of this paper is that in certain cases the corresponding algebra of Hecke operators turns out to be a homomorphic image of the quantum toroidal algebra. The latter is a quantization, in the spirit of Drinfeld [Dr 1] and Jimbo [Ji], of the universal enveloping algebra of the universal central extension of a "double-loop" Lie algebra. This yields, in particular, a new geometric construction of affine quantum groups of types $A^{(1)}, D^{(1)}$ and $E^{(1)}$ in terms of Hecke operators for an elliptic surface.

Received January 30, 1995.

The second author was partially supported by an NSF grant and an Alfred P. Sloan Research Fellowship. 
Our approach was motivated in part by a relation between instantons on ALE-spaces and affine Lie algebras discovered by Nakajima [Na 1] (which was in its turn motivated by Lusztig's construction [Lu] of quantum groups in terms of quivers and the Lagrangian construction of [Gi]). Nakajima's results were also used by Vafa and Witten [VW] in verifying a special case of their $S$-duality conjecture. We believe that there is in fact a very close connection between the Langlands' reciprocity for an algebraic surface and the $S$-duality conjecture for the underlying real 4 -manifold. Suffices it to say that the interplay between a reductive group $G$ and the Langlands dual group $G^{\vee}$ is quite essential in both cases.

Proofs of the results announced in this paper will appear elsewhere.

\section{Hecke operators and Satake isomorphism}

We recall here the well-known interpretation of Hecke operators in terms of moduli spaces (see, e.g., [Dr 3]).

Let $C$ be a complete smooth curve over a finite field $\mathbb{F}_{p}$ and $x \in C$ an $\mathbb{F}_{p}$-rational point. Let $\mathcal{M}$ be the discrete set of isomorphism classes of $\mathbb{F}_{p}$-rational vector bundles on $C$ of rank $d$. Given a vector bundle $V \in \mathcal{M}$ and $W \subset V_{x}$, a linear subspace in the fiber of $V$ at $x$, let $\mathcal{A}_{x}(V, W)$ denote the subsheaf of $V$ consisting of sections whose value at $x$ is contained in $W$. This sheaf is again locally free, hence, can be regarded as a new vector bundle on $C$, called the elementary transformation of $V$. Let $\mathbb{C}[\mathcal{M}]$ be the vector space of $\mathbb{C}$-valued functions on $\mathcal{M}$ with finite support. For any $k=1, \ldots, d$, the following sum is finite, hence defines a Hecke operator $T_{x, k}: \mathbb{C}[\mathcal{M}] \rightarrow \mathbb{C}[\mathcal{M}]$ by

$$
T_{x, k}(f)(V)=\sum_{\left\{W \subset V_{x} \mid \operatorname{dim} W=d-k\right\}} f\left(\mathcal{A}_{x}(V, W)\right) .
$$

Let $\mathbf{H}_{x}$ denote the $\mathbb{C}$-subalgebra of linear endomorphisms of the vector space $\mathbb{C}[\mathcal{M}]$ generated by the operators $T_{x, k}, k=1, \ldots, d$.

Let $\mathbb{C}[G]^{G}$ denote the algebra of the regular class functions on the complex algebraic group $G=G L_{d}(\mathbb{C})$. The classical theorem of Satake, combined with the fact that the natural $\mathbf{H}_{x}$-action makes $\mathbb{C}[\mathcal{M}]$ a faithful $\mathbf{H}_{x}$-module, yields the following version of the Satake isomorphism.

Theorem 1.1. There is a natural algebra isomorphism:

$$
\mathbb{C}[G]^{G} \simeq \mathbf{H}_{x} .
$$




\section{Hecke operators for algebraic surfaces}

We construct here certain analogues of Hecke operators for algebraic surfaces. These operators are associated to curves rather than to points on the surface.

Let $S$ be a smooth projective surface over a finite field $\mathbb{F}_{p}$, and $C \subset S$ a curve (i.e., possibly non-reduced subscheme, locally given by one equation defined over $\mathbb{F}_{p}$ ). Write $\mathcal{M}$ for the discrete set of isomorphism classes of $d$ dimensional algebraic vector bundles on $S$. Given $\left.W \subset V\right|_{C}$, a subbundle (i.e., subsheaf which is locally a direct summand) $W$ in the restriction of $V$ to $C$, we introduce the subsheaf $\mathcal{A}_{C}(V, W)$ in $V$ to be formed by the sections of $s \in V$ such that $\left.s\right|_{C} \in W$. This sheaf is locally free and, hence, can be regarded as a new vector bundle on $S$, see [Ma].

Let $\mathcal{O}_{S}(-C)$ be the invertible sheaf on $S$ formed by functions vanishing at $C$, let $\mathcal{O}_{S}(C)$ be its dual, and $\mathcal{O}_{C}(-C)$ the (scheme theoretic) restriction of $\mathcal{O}_{S}(-C)$ to $C$. Let $\mathbb{C}[\mathcal{M}]$ denote the vector space of complex functions on $\mathcal{M}$ with finite support. To any vector bundle $L$ on $C$ we associate two Hecke operators $T_{L}, T_{L}^{*}: \mathbb{C}[\mathcal{M}] \rightarrow \mathbb{C}[\mathcal{M}]$ given by the following formulas

$$
\begin{gathered}
\left(T_{L} f\right)(V)=\sum_{\left\{\left.W \subset V\right|_{C} \text { such that }\left(\left.V\right|_{C}\right) / W \simeq L\right\}} f\left(\mathcal{A}_{C}(V, W)\right) \\
\left(T_{L}^{*} f\right)(V)=\sum_{\left\{\left.W \subset V\right|_{C} \text { such that } W \simeq L \otimes \mathcal{O}_{C}(-C)\right\}} f\left(\mathcal{A}_{C}(V, W) \otimes \mathcal{O}_{S}(C)\right),
\end{gathered}
$$

Assume now that $C \simeq \mathbb{P}^{1}$ is a projective line. Let $\mathcal{M}_{C} \subset \mathcal{M}$ be the set of those vector bundles $V$ whose restrictions to $C$ have the form

$$
\left.V\right|_{C} \simeq \mathcal{O}_{\mathbb{P}^{1}}(1)^{\oplus a(V)} \oplus \mathcal{O}_{\mathbb{P}^{1}}(0)^{\oplus b(V)} \oplus \mathcal{O}_{\mathbb{P}^{1}}(-1)^{\oplus c(V)}, \quad a(V), b(V), c(V) \in \mathbb{N}
$$

Given $V \in \mathcal{M}_{C}$ and $\left.W \subset V\right|_{C}$, a subbundle with quotient isomorphic to $\mathcal{O}_{\mathbb{P} 1}(-1)$, put $V^{\prime}=\mathcal{A}_{C}(V, W)$. Then $V^{\prime}$ is again in $\mathcal{M}_{C}$, and we have

$$
a\left(V^{\prime}\right)=a(V)+1 \quad, \quad b\left(V^{\prime}\right)=b(V) \quad, \quad c\left(V^{\prime}\right)=c(V)-1
$$

Therefore, the operators $T_{\mathcal{O}_{C}(-1)}$ and $T_{\mathcal{O}_{C}(-1)}^{*}$ are well defined on the vector space $\mathbb{C}\left[\mathcal{M}_{C}\right]$, viewed as the subspace of functions on $\mathcal{M}$ vanishing off $\mathcal{M}_{C}$. We introduce the following modified operators $\mathbf{E}, \mathbf{F}$ on $\mathbb{C}\left[\mathcal{M}_{C}\right]$ :

$$
(\mathbf{E} f)(V)=p^{-c(V) / 2} \cdot\left(T_{\mathcal{O}_{C}(-1)}^{*} f\right)(V), \quad(\mathbf{F} f)(V)=p^{-a(V) / 2} \cdot\left(T_{\mathcal{O}_{C}(-1)} f\right)(V)
$$

Define also the third operator $\mathbf{K}: \mathbb{C}\left[\mathcal{M}_{C}\right] \rightarrow \mathbb{C}\left[\mathcal{M}_{C}\right]$ by the formula

$$
(\mathbf{K} f)(V)=p^{(a(V)-c(V)) / 2} \cdot f(V) .
$$


Recall now that to any generalized Cartan matrix one can associate a Kac-Moody Lie algebra g. Furthermore, Drinfeld [Dr 1] and Jimbo [Ji] have defined a $q$-deformation, $\mathbf{U}_{\mathbf{q}}(\mathfrak{g})$, of the universal enveloping algebra of $\mathfrak{g}$, the so-called quantized universal enveloping algebra.

The following crucial observation is at the origin of the relationship between quantum groups and Hecke operators.

Proposition 2.3. If curve $C$ is isomorphic to $\mathbb{P}^{1}$ and has self-intersection $(-2)$ in $S$, then the operators $\mathbf{E}, \mathbf{F}$ and $\mathbf{K}$ satisfy the commutation relations of the standard Chevalley generators of the algebra $\mathbf{U}_{\mathbf{q}}\left(\mathfrak{s l}_{2}\right)$, specialized at $\mathbf{q}=p^{1 / 2}$.

Remark. An analogue of the proposition still holds if the projective line $C=\mathbb{P}^{1} \subset S$ has an arbitrary negative self-intersection $-m<0$. Then, one should consider vector bundles $V$ on $S$ such that $\left.V\right|_{C}$ is a direct sum of several copies of the sheaves $\mathcal{O}_{\mathbb{p} 1}(0), \ldots, \mathcal{O}_{\mathbb{p} 1}(m)$.

Observe next that decomposition of a vector bundle $V$ on $\mathbb{P}^{1}$ into a direct sum of line bundles, like (2.1), is not canonical in general. What is only canonical is the filtration by subbundles $0=\ldots \subseteq F_{j-1} V \subseteq F_{j} V \subseteq$ $F_{j+1} V \subseteq \ldots=V$ such that, for any $j \in \mathbb{Z}$, the quotient $F_{j} V / F_{j-1} V$ is the direct sum of several copies of $\mathcal{O}_{\mathbb{p} 1}(-j)$. The filtration $F_{\bullet} V$ measures the "unstability" of the vector bundle $V$ and is called the Harder-Narasimhan filtration. Thus, for any point $x \in \mathbb{P}^{1}$, the fiber $V_{x}$ of $V$ acquires a canonical finite increasing filtration $F_{\bullet} V_{x}$.

We now turn to a more general case where $C \subset S$ is a reducible algebraic curve with smooth irreducible components $C_{i}, i \in \Delta$, isomorphic to $\mathbb{P}^{1}$ each. Assume in addition that different components intersect each other transversely, and each $C_{i}$ has self-intersection $(-2)$. Thus, the intersection matrix of the irreducible components of $C$ is the opposite of a generalized Cartan matrix, $\|a(C)\|$.

Call a vector bundle $V$ on the curve $C$ admissible if the following conditions hold :

(i) For each irreducible component $C_{i}$, the restriction $\left.V\right|_{C_{i}}$ has a direct sum decomposition like (2.1).

(ii) For any intersection point $x$ of two adjacent components $C_{i}$ and $C_{j}$, the two flags in the fiber at $x: \quad\left(0=F_{-2} V_{x} \subseteq F_{-1} V_{x} \subseteq\right.$ $\left.F_{0} V_{x} \subseteq F_{1} V_{x}=V_{x}\right)$ and $\left(0=F_{-2}^{\prime} V_{x} \subseteq F_{-1}^{\prime} V_{x} \subseteq F_{0}^{\prime} V_{x} \subseteq F_{1}^{\prime} V_{x}=\right.$ $V_{x}$ ) induced by the canonical Harder-Narasimhan filtrations on $\left.V\right|_{C_{i}}$ and $\left.V\right|_{C_{j}}$ are in generic relative position.

Fix an integer $d \geq 1$ and let $\mathcal{M}_{C}$ be the moduli space of $d$-dimensional algebraic vector bundles on the surface $S$ that have admissible restriction to $C$. For any $V \in \mathcal{M}_{C}, i \in \Delta$ and a subbundle $\left.W \subset V\right|_{C_{i}}$ such 
that $\left(\left.V\right|_{C_{i}}\right) / W \simeq \mathcal{O}_{C_{i}}(-1)$, the vector bundle $\mathcal{A}_{C_{i}}(V, W)$ is again in $\mathcal{M}_{C}$. Thus, the operators $T_{\mathcal{O}_{C_{i}}(-1)}$ and $T_{\mathcal{O}_{C_{i}}(-1)}^{*}$ are well-defined on $\mathbb{C}\left[\mathcal{M}_{C}\right]$ and, for each $i \in \Delta$, we introduce the operators $\mathbf{E}_{i}, \mathbf{F}_{i}, \mathbf{K}_{i}$, defined as in (2.2), but with respect to the corresponding component $C_{i}$. The following result motivated by Nakajima [Na 1] gives way to an interpretation of any quantized Kac-Moody algebra $\mathbf{U}_{\mathbf{q}}(\mathfrak{g})$ in terms of Hecke operators.

Theorem 2.4. Let $\mathfrak{g}$ be the Kac-Moody algebra whose Cartan matrix is $\|a(C)\|$, the opposite of the intersection matrix. Then, the operators

$$
\left\{\mathbf{E}_{i}, \mathbf{F}_{i}, \mathbf{K}_{i} \in \operatorname{End} \mathbb{C}\left[\mathcal{M}_{C}\right], i \in \Delta\right\}
$$

satisfy the quantum Serre relations for the standard Chevalley generators of the quantum algebra $\mathbf{U}_{\mathbf{q}}(\mathfrak{g})$, specialized at $\mathbf{q}=p^{1 / 2}$.

Remarks. (i) During the preparation of this paper we received Nakajima's preprint [ $\mathrm{Na} 2]$ where a similar result is proved in the setup of instantons on an ALE-space (=desingularization of $\mathbb{C}^{2} / \Gamma$ for a finite subgroup $\Gamma \subset S L_{2}(\mathbb{C})$ ) instead of algebraic vector bundles on a compact algebraic surface. Nakajima's proof is based on an explicit ADHM-type construction of instantons on an ALE-space in terms of a linear algebra data. There is no ADHM-type construction for an arbitrary surface, of course. Our approach is different. We verify the Serre relations directly using a local geometry of vector bundles on a small neighborhood of the curve $C \subset S$. Somewhat miraculously, the actual computation turns out to be quite similar to the computation involving the Grassmannians of $k$-planes in $\left(\mathbb{F}_{p}\right)^{d}$, carried out in [BLM].

(ii) There are many interesting example of configurations of $\mathbb{P}^{1}$ 's on surfaces other than ALE-spaces. Take, for instance, an Enriques surface $S$ containing a configuration with intersection matrix of affine type $E_{8}^{(1)}$, see [Ko]. The double cover $S^{\prime}$ of $S$ is a K3-surface with two such configurations not intersecting each other. Thus, we get an action on an appropriate space $\mathbb{C}\left[\mathcal{M}_{C}\right]$ of two commuting copies of the quantum algebra $\mathbf{U}_{\mathbf{q}}(\mathfrak{g})$, where $\mathfrak{g}$ is the affine Lie algebra of type $E_{8}^{(1)}$. We would like to thank R. Bezrukavnikov for pointing out this example.

We now turn to Langlands reciprocity. Let $S$ be an arbitrary smooth projective surface over $\mathbb{F}_{p}$, and $C \subset S$ an algebraic curve. Let $\mathcal{M}$ be the set of $\mathbb{F}_{p}$-rational algebraic vector bundles on $S$ of rank $d$, and $\mathcal{M}^{\circ} \subset \mathcal{M}$ the subset of all vector bundles that are trivial on $S \backslash C$. Choose an integer $l$ prime to $p$, and let $\mathcal{L}_{o c_{C}}$ be the set of $l$-adic local systems on $C$ of rank $d$. Then we conjecture the following : 
Conjecture 2.5 (Langlands conjecture for surfaces).

(i) For any pair $(S, C)$, there is a natural algebra homomorphism

$$
\mathbb{C}\left[\mathcal{L}_{o c_{C}}\right] \rightarrow \operatorname{End} \mathbb{C}\left[\mathcal{M}^{\circ}\right] \quad, \quad f \mapsto T_{f}
$$

(ii) To any d-dimensional local system $\phi$ on $S$, one can associate an "automorphic" function $F_{\phi} \in \mathbb{C}\left[\mathcal{M}^{\circ}\right]$ such that we have

$$
T_{f}\left(F_{\phi}\right)=f\left(\left.\phi\right|_{C}\right) \cdot F_{\phi} \quad \text { for every } f \in \mathbb{C}\left[\mathcal{L}_{o c_{C}}\right] .
$$

Assume next that $S$ is a complex smooth projective surface with a holomorphic symplectic structure (e.g., a $K 3$-surface) and $C \subset S$ a connected algebraic curve such that $C \cdot C \leq 0$ (so $C$ is either a rational or an elliptic curve). Let $\mathcal{M}$ denote the moduli stack of algebraic (not necessarily stable) vector bundles with trivial determinant on $S$, and let $\mathcal{M}^{\circ}$ denote the subspace of the vector bundles trivial on $S \backslash C$. The symplectic structure on $S$ induces a canonical symplectic structure on $\mathcal{M}$. The following conjecture is motivated, in part, by the relationship between 2-dimensional field theory and 3-dimensional Chern-Simons theory (see Witten [Wi]).

Conjecture 2.6. $\mathcal{M}^{\circ}$ is a Lagrangian subvariety in $\mathcal{M}$.

\section{Quantum toroidal algebras}

Given a complex semisimple Lie algebra $\mathfrak{g}$, write $\mathfrak{g}(z, u)$ for the Lie algebra of all (Laurent) polynomial maps $\mathbb{C}^{*} \times \mathbb{C}^{*} \rightarrow \mathfrak{g}$ with the pointwise bracket. This 'double-loop' Lie algebra has a universal central extension by the infinite dimensional vector space $\Omega^{1} / d \Omega^{0}$, where $\Omega^{i}$ stands for polynomial $i$-forms on $\mathbb{C}^{*} \times \mathbb{C}^{*}$. The extension is denoted $\mathfrak{g}_{\text {tor }}$ and is called the toroidal Lie algebra associated to $\mathfrak{g}$. A description of toroidal algebras in terms of generators and relations was first given in [MRY]. We now sketch a construction of a quantum deformation of the universal enveloping algebra $U\left(\mathfrak{g}_{\text {tor }}\right)$.

Assume first that $\mathfrak{g}=\mathfrak{s l}_{2}$. The quantum toroidal algebra, $\mathbf{U}_{\mathbf{q}}\left(\mathfrak{s l}_{2, \text { tor }}\right)$, is a $\mathbb{C}(q)$-algebra on generators

$$
E_{0}, E_{1}, E_{2}, F_{0}, F_{1}, F_{2}, K_{0}^{ \pm 1}, K_{1}^{ \pm 1}, K_{2}^{ \pm 1},
$$

that satisfy, in particular, the following relations

$$
\begin{array}{cc}
K_{i} \cdot K_{i}^{-1}=K_{i}^{-1} \cdot K_{i}=1 \quad & K_{i} \cdot K_{j}=K_{j} \cdot K_{i}, \\
K_{i} \cdot E_{j} \cdot K_{i}^{-1}=q^{a_{i j}} \cdot E_{j} \quad, & K_{i} \cdot F_{j} \cdot K_{i}^{-1}=q^{-a_{i j}} \cdot F_{j}, \\
E_{2} \cdot E_{0}=q^{-2} \cdot E_{0} \cdot E_{2} \quad, & F_{2} \cdot F_{0}=q^{-2} \cdot F_{0} \cdot F_{2},
\end{array}
$$


involving the 'double-extended' Cartan matrix:

$$
\left\|a_{i, j}\right\|=\left(\begin{array}{rrr}
2 & -2 & 2 \\
-2 & 2 & -2 \\
2 & -2 & 2
\end{array}\right) \quad, \quad i, j=0,1,2
$$

Further, if $|j-i| \leq 1$ we have :

$$
\left[E_{i}, F_{j}\right]=\delta_{i j} \frac{K_{i}-K_{i}^{-1}}{q-q^{-1}}
$$

and if $|j-i|=1$ the following Serre-type relations hold:

$\sum_{m=0}^{3}(-1)^{m}\left[\begin{array}{c}3 \\ m\end{array}\right] \cdot E_{i}^{3-m} \cdot E_{j} \cdot E_{i}^{m}=0, \quad \sum_{m=0}^{3}(-1)^{m}\left[\begin{array}{c}3 \\ m\end{array}\right] \cdot F_{i}^{3-m} \cdot F_{j} \cdot F_{i}^{m}=0$,

where $\left[\begin{array}{l}i \\ j\end{array}\right]$ denote $q$-binomial coefficients. In addition, whenever $j-i= \pm 2$ we have (put " \pm " respectively):

$$
\begin{aligned}
& \sum_{m=0}^{3}\left(-q^{2}\right)^{ \pm m}\left[\begin{array}{c}
3 \\
m
\end{array}\right] \cdot E_{i}^{3-m} \cdot F_{j} \cdot E_{i}^{m}=0 \\
& \sum_{m=0}^{3}\left(-q^{2}\right)^{ \pm m}\left[\begin{array}{c}
3 \\
m
\end{array}\right] \cdot F_{i}^{3-m} \cdot E_{j} \cdot F_{i}^{m}=0 .
\end{aligned}
$$

There are infinitely many other relations of higher order, however. Therefore, for general semisimple Lie algebras, it will be more convenient to use an alternative approach similar to the "loop-like" realization of quantum affine algebras, introduced by Drinfeld [Dr 2].

Thus, given a semisimple Lie algebra $\mathfrak{g}$, let $\left\|a_{\alpha \beta}\right\|_{(\alpha, \beta=0,1, \ldots, r)}$ be the extended (i.e., affine) Cartan matrix associated with $\mathfrak{g}$. This matrix is symmetrizable, that is, there are mutually prime integers $d_{0}, d_{1}, \ldots, d_{r}$ such that the matrix with the entries $b_{\alpha \beta}=d_{\alpha} \cdot a_{\alpha \beta},(\alpha, \beta=0,1, \ldots, r)$ is symmetric. Thus, we have two $(r+1) \times(r+1)$-matrices $\left\|a_{\alpha \beta}\right\|$ and $\left\|b_{\alpha \beta}\right\|$.

Let $A$ be the free associative $\mathbb{C}\left[q, q^{-1}, C, C^{-1}\right]$-algebra on generators $E_{\alpha, k}, F_{\alpha, k}, H_{\alpha, l}, K_{\alpha}^{ \pm 1},(\alpha \in[0, r], k, l \in \mathbb{Z}, l \neq 0)$. Form the following generating functions :

$$
\begin{gathered}
K_{\alpha}^{ \pm}(z)=K_{\alpha}^{ \pm} \cdot \exp \left(\left(q-q^{-1}\right) \sum_{k \geq 1} H_{\alpha}( \pm k) \cdot z^{\mp k}\right) \in A\left[\left[z^{\mp 1}\right]\right], \\
E_{\alpha}(z)=\sum_{k=-\infty}^{\infty} E_{\alpha, k} \cdot z^{-k}, F_{\alpha}(z)=\sum_{k=-\infty}^{\infty} F_{\alpha, k} \cdot z^{-k} \in A\left[\left[z, z^{-1}\right]\right],
\end{gathered}
$$


We define the quantum toroidal algebra, $\mathbf{U}_{\mathbf{q}}\left(\mathfrak{g}_{\text {tor }}\right)$, to be the quotient of the free algebra $A$ modulo a number of relations. These are most conveniently expressed in terms of the rational function $\theta_{m}(z)=\frac{q^{m} \cdot z-1}{z-q^{m}}$ and the generating functions $K_{\alpha}^{ \pm}(z), E_{\alpha}(z), F_{\alpha}(z)$ above as follows (cf. [Dr 2]) :

$$
\begin{aligned}
K_{\alpha}^{ \pm}(z) \cdot K_{\beta}^{ \pm}(w) & =K_{\beta}^{ \pm}(w) \cdot K_{\alpha}^{ \pm}(z), \\
\theta_{b_{\alpha, \beta}}\left(C^{-2} \cdot \frac{z}{w}\right) \cdot K_{\alpha}^{+}(z) \cdot K_{\beta}^{-}(w) & =\theta_{b_{\alpha, \beta}}\left(C^{2} \cdot \frac{z}{w}\right) \cdot K_{\beta}^{-}(w) \cdot K_{\alpha}^{+}(z), \\
K_{\alpha}^{ \pm}(z) \cdot E_{\beta}(w) & =\theta_{b_{\alpha, \beta}}\left(C^{ \pm 1} \cdot \frac{z}{w}\right) \cdot E_{\beta}(w) \cdot K_{\alpha}^{ \pm}(z), \\
K_{\alpha}^{ \pm}(z) \cdot F_{\beta}(w) & =\theta_{-b_{\alpha, \beta}}\left(C^{\mp 1} \cdot \frac{z}{w}\right) \cdot F_{\beta}(w) \cdot K_{\alpha}^{ \pm}(z), \\
\left(q-q^{-1}\right)\left[E_{\alpha}(z), F_{\beta}(w)\right] & = \\
\delta_{\alpha, \beta}\left(\delta\left(C^{-2} \cdot \frac{z}{w}\right) \cdot K_{\alpha}^{+}(C \cdot w)\right. & \left.-\delta\left(C^{2} \cdot \frac{z}{w}\right) \cdot K_{\alpha}^{-}(C \cdot z)\right), \\
E_{\alpha}(z) \cdot E_{\beta}(w) & =\theta_{b_{\alpha, \beta}}\left(\frac{z}{w}\right) \cdot E_{\beta}(w) \cdot E_{\alpha}(z), \\
F_{\alpha}(z) \cdot F_{\beta}(w) & =\theta_{-b_{\alpha, \beta}}\left(\frac{z}{w}\right) \cdot F_{\beta}(w) \cdot F_{\alpha}(z),
\end{aligned}
$$

and the symmetrizations with respect to $z_{1}, z_{2}, \ldots, z_{1-a_{\alpha, \beta}}$ of each of the following two expressions vanish :

$$
\begin{gathered}
\sum_{i=0}^{1-a_{\alpha, \beta}}(-1)^{i}\left[\begin{array}{c}
1-a_{\alpha, \beta} \\
i
\end{array}\right] E_{\alpha}\left(z_{1}\right) \cdots E_{\alpha}\left(z_{i}\right) E_{\beta}(w) E_{\alpha}\left(z_{i+1}\right) \cdots E_{\alpha}\left(z_{1-a_{\alpha, \beta}}\right) \\
\sum_{i=0}^{1-a_{\alpha, \beta}}(-1)^{i}\left[\begin{array}{c}
1-a_{\alpha, \beta} \\
i
\end{array}\right] F_{\alpha}\left(z_{1}\right) \cdots F_{\alpha}\left(z_{i}\right) F_{\beta}(w) F_{\alpha}\left(z_{i+1}\right) \cdots F_{\alpha}\left(z_{1-a_{\alpha, \beta}}\right)
\end{gathered}
$$

The specialization of the quantum toroidal algebra at $q=1$ reduces to the universal enveloping algebra $U\left(\mathfrak{g}_{\text {tor }}\right)$. Furthermore, the quantum toroidal algebra has the natural structure of a Hopf algebra.

The double-loop Lie algebra $\mathfrak{g}(z, w)$ has an obvious automorphism which switches the two variables $z$ and $w$. The following "quantized analogue" of this observation, motivated by [Ch], clarifies the structure of $\mathbf{U}_{\mathbf{q}}\left(\mathfrak{g}_{\text {tor }}\right)$.

Proposition 3.1. Let $U_{1} \subset \mathbf{U}_{\mathbf{q}}\left(\mathfrak{g}_{\text {tor }}\right)$ be the subalgebra generated by the elements $E_{\alpha, 0}, F_{\alpha, 0}, K_{\alpha}^{ \pm}, \alpha=0,1, \ldots r$, and let $U_{2} \subset \mathbf{U}_{\mathbf{q}}\left(\mathfrak{g}_{\text {tor }}\right)$ be the subalgebra generated by all the coefficients of the generating functions $E_{\alpha}(z)$, $F_{\alpha}(z), K_{\alpha}^{ \pm}(z), \alpha=1,2 \ldots r$ (note that $\alpha \neq 0$ ). Then we have

(i) The algebras $U_{1}$ and $U_{2}$ are both isomorphic to the quantum enveloping algebra with affine Cartan matrix $\left\|a_{\alpha \beta}\right\|_{\alpha, \beta=0, \ldots, r}$. Moreover, $U_{1} \cap U_{2}=\mathbf{U}_{\mathbf{q}}(\mathfrak{g})$, is the quantum enveloping algebra with finite Cartan matrix $\left\|a_{\alpha \beta}\right\|_{\alpha, \beta=1, \ldots, r}$.

(ii) There is an automorphism of the algebra $\mathbf{U}_{\mathbf{q}}\left(\mathfrak{g}_{\text {tor }}\right)$ taking $U_{1}$ to $U_{2}$. 


\section{Elliptic surface case}

The considerations of section 2 become much more concrete for elliptic surfaces. Thus, we fix in this section a smooth projective surface $S$, a smooth curve $X$, and a morphism $\pi: S \rightarrow X$ whose general fibre is an elliptic curve. Let $o \in X$ be a point such that the fiber $C=\pi^{-1}(o)$ is not isomorphic to an elliptic curve. Such a fiber $C$ is known to be a connected rational curve. Call $C$ a non-multiple special fiber if the multiplicities of the irreducible components of $C$ are relatively prime. The local classification of non-multiple special fibers in elliptic surfaces, due to Kodaira [Ko] and Néron [Né], says that except for a few cases, they are all of the type considered in section 2. More precisely, if we exclude the special fibers of type $A, C_{1}, C_{2}$ and $C_{3}$, as given in table [Ta, p.46], the irreducible components of a non-multiple special fiber, $C$, are isomorphic to $\mathbb{P}^{1}$ each and their intersection matrix is the opposite of the Cartan matrix $\|a(C)\|$ of an affine root system. Moreover, the correspondence $\|a(C)\| \leftrightarrow C$ sets up a bijection between the affine root systems of type $A^{(1)}, D^{(1)}, E^{(1)}$ and the possible types of non-multiple special fibers (different from $A, C_{1}, C_{2}$ and $\left.C_{3}\right)$. Let $\mathfrak{g}_{C}$ denote the affine Lie algebra associated to $\|a(C)\|$. We thus obtain from theorem 2.4 the following result:

Theorem 4.1. The assignment of the algebra generated by the Hecke operators $\left\{\mathbf{E}_{i}, \mathbf{F}_{i}, \mathbf{K}_{i} \in\right.$ End $\left.\mathbb{C}\left[\mathcal{M}_{C}\right], i \in \Delta\right\}$, to a non-multiple special fiber of an elliptic surface yields a bijective correspondence between the possible types of a special fiber and the quantum affine algebras of type $A^{(1)}, D^{(1)}$ and $E^{(1)}$.

It is likely that there is a similar interpretation of elliptic algebras (see [GKV]) in terms of Hecke operators which correspond to a general nonsingular fiber of an elliptic surface. When the fiber degenerates to a special one, the elliptic algebra should degenerate to a quantum affine algebra.

We now extend theorem 2.4 to quantum toroidal algebras. To that end, let $C^{\circ} \subset S$ be the curve obtained from a non-multiple special fiber $C$ of the elliptic surface $S$ by removing one geometric component in such a way that the intersection matrix of the set $\Delta^{\circ} \subset \Delta$ of components of $C^{\circ}$ is of the corresponding (finite) type $A, D, E$. We view $C^{\circ}$ as a scheme (scheme structure is induced by that on $\pi^{-1}(o)$ ), and let $n_{i}, i \in \Delta^{\circ}$, denote the multiplicities of the irreducible components of $C^{\circ}$. Observe that the integers $n_{i}$ are the coordinates of the maximal root of the root system $\Delta^{\circ}$ in the basis of simple roots.

In the setup of $\S 2$ we introduce two additional operators $\mathbf{E}_{0}, \mathbf{F}_{0}$ corresponding to the reducible curve $C^{\circ}$ as a whole by the formulas $\mathbf{E}_{0}=T_{\mathcal{O}_{C^{\circ}}}$, 
$\mathbf{F}_{0}=T_{\mathcal{O}^{\circ}}^{*}$. Recall $d=r k V$, and put

$$
\left(\mathbf{K}_{0} f\right)(V)=p^{\left(d-\sum_{i \in \Delta^{\circ}} n_{i}\left(a_{i}(V)-c_{i}(V)\right)\right) / 2} \cdot f(V)
$$

Theorem 4.2. Let $\mathfrak{g}_{\text {tor }}$ be the toroidal algebra associated with the affine Cartan matrix $\|a(C)\|$, the intersection matrix. Then, there is an algebra homomorphism $\left.\mathbf{U}_{\mathbf{q}}\left(\mathfrak{g}_{\text {tor }}\right)\right|_{\mathbf{q}=p^{1 / 2}} \rightarrow$ End $\mathbb{C}\left[\mathcal{M}_{C}\right]$. The image of this homomorphism is the algebra generated by the Hecke operators $\left\{\mathbf{E}_{i}, \mathbf{F}_{i}, \mathbf{K}_{i} \in\right.$ End $\left.\mathbb{C}\left[\mathcal{M}_{C}\right], i \in \Delta \cup\{0\}\right\}$.

Remark. The two subalgebras generated by the elements

$$
\left\{\mathbf{E}_{i}, \mathbf{F}_{i}, \mathbf{K}_{i}, i \in \Delta\right\} \text { and }\left\{\mathbf{E}_{i}, \mathbf{F}_{i}, \mathbf{K}_{i}, i \in \Delta^{\circ} \cup\{0\}\right\}
$$

respectively correspond, via the theorem, to the subalgebras $U_{1}$ and $U_{2}$ introduced in Proposition 3.1.

\section{5. $K$-theoretic construction of the toroidal algebra for $\mathfrak{s l}_{n}$}

Fix another positive integer $n$. In the special case $\mathfrak{g}=\mathfrak{s l}_{n}$ the quantum toroidal algebra $\mathbf{U}_{\mathbf{q}}\left(\mathfrak{s l}_{n, \text { tor }}\right)$ has an alternative definition. It is based on the well-known realization of the affine Lie algebra of type $A_{n}^{(1)}$ as the subalgebra of " $n$-periodic" elements in the affine Lie algebra $\mathfrak{g}_{\infty}$ of type $A_{\infty}^{(1)}$. Taking loops in both algebras yields an imbedding of the toroidal Lie algebra into $\hat{\mathfrak{g}}_{\infty}$, the central extension of the loop algebra on $\mathfrak{g}_{\infty}$. This way, the algebra $\mathbf{U}_{\mathbf{q}}\left(\mathfrak{s l}_{n, \text { tor }}\right)$ can be viewed as the subalgebra of " $n$-periodic" elements of $\mathbf{U}_{\mathbf{q}}\left(\hat{\mathfrak{g}}_{\infty}\right)$, the quantized enveloping algebra associated (in the sense of [Dr 2]) with the Lie algebra $\hat{\mathfrak{g}}_{\infty}$.

It is useful to "enlarge" the algebra $\mathbf{U}_{\mathbf{q}}\left(\mathfrak{s l}_{n, \text { tor }}\right)$ slightly and introduce an extended quantum toroidal algebra $\mathbf{U}$ with a central extra-generator $D$ as follows. Let $\left\|a_{\alpha \beta}\right\|$ be the affine Cartan matrix of type $A_{n-1}^{(1)}$, and $\left\|m_{\alpha \beta}\right\|$ the $n \times n$-matrix given by $m_{\alpha \beta}=\delta_{\alpha, \beta+1}-\delta_{\alpha+1, \beta}$, where $\delta$ is Kronecker's $\delta$ and $\alpha, \beta \in \mathbb{Z} / n \mathbb{Z}$. Let $\mathbf{U}$ be quotient of the free associative $\mathbb{C}\left[q, q^{-1}\right.$, $C, C^{-1}, D, D^{-1}$-algebra on the same set of generators as in $\S 3$ modulo the 
modified relations

$$
\begin{aligned}
K_{\alpha}^{ \pm}(z) \cdot K_{\beta}^{ \pm}(w) & =K_{\beta}^{ \pm}(w) \cdot K_{\alpha}^{ \pm}(z) \\
\theta_{a_{\alpha, \beta}}\left(C^{-2} D^{m_{\alpha \beta}} \cdot \frac{z}{w}\right) \cdot K_{\alpha}^{+}(z) & \cdot K_{\beta}^{-}(w) \\
& =\theta_{a_{\alpha, \beta}}\left(C^{2} D^{m_{\alpha \beta}} \cdot \frac{z}{w}\right) \cdot K_{\beta}^{-}(w) \cdot K_{\alpha}^{+}(z), \\
K_{\alpha}^{ \pm}(z) \cdot E_{\beta}(w) & =\theta_{a_{\alpha, \beta}}\left(C^{ \pm 1} D^{m_{\alpha \beta}} \cdot \frac{z}{w}\right) \cdot E_{\beta}(w) \cdot K_{\alpha}^{ \pm}(z), \\
K_{\alpha}^{ \pm}(z) \cdot F_{\beta}(w) & =\theta_{-a_{\alpha, \beta}}\left(C^{\mp 1} D^{m_{\alpha \beta}} \cdot \frac{z}{w}\right) \cdot F_{\beta}(w) \cdot K_{\alpha}^{ \pm}(z), \\
\left(q-q^{-1}\right)\left[E_{\alpha}(z), F_{\beta}(w)\right] & = \\
\delta_{\alpha, \beta}\left(\delta\left(C^{-2} \cdot \frac{z}{w}\right) \cdot K_{\alpha}^{+}(C \cdot w)\right. & \left.-\delta\left(C^{2} \cdot \frac{z}{w}\right) \cdot K_{\alpha}^{-}(C \cdot z)\right) \\
E_{\alpha}(z) \cdot E_{\beta}(w) & =\theta_{a_{\alpha, \beta}}\left(D^{m_{\alpha \beta}} \frac{z}{w}\right) \cdot E_{\beta}(w) \cdot E_{\alpha}(z) \\
F_{\alpha}(z) \cdot F_{\beta}(w) & =\theta_{-a_{\alpha, \beta}}\left(D^{m_{\alpha \beta}} \frac{z}{w}\right) \cdot F_{\beta}(w) \cdot F_{\alpha}(z)
\end{aligned}
$$

and same Serre-type relations as in $\S 3$.

We produce below a geometric construction of the extended quantum toroidal algebra $\mathbf{U}$. To that end, let $\mathbb{K}=\mathbb{C}((z))$ be the field of Laurent formal power series. Given an integer $d \geq 1$, write $\mathbb{K}^{d}$ for the $d$-dimensional coordinate vector space over $\mathbb{K}$. By a lattice we mean a rank $d$ free $\mathbb{C}[[z]]$ submodule in $\mathbb{K}^{d}$.

Following an idea of Beilinson-Lusztig-MacPherson [BLM] in the finite case and its affine version introduced in [GV 1], let $\mathcal{B}$ be the "affine $n$ periodic partial flag variety" consisting of the sequences of lattices in $\mathbb{K}^{d}$ of the form

$F=\left(\ldots \subseteq F_{i-1} \subseteq F_{i} \subseteq F_{i+1} \subseteq \ldots\right)$ such that $F_{i+n}=z^{-1} \cdot F_{i}, \forall i \in \mathbb{Z}$.

Let $G(\mathbb{K})=G L_{d}(\mathbb{K})$ be the group of invertible $\mathbb{K}$-valued $(d \times d)$-matrices. The natural $G(\mathbb{K})$-action on the set of lattices induces a $G(\mathbb{K})$-action on $\mathcal{B}$ and the diagonal $G(\mathbb{K})$-action on $\mathcal{B} \times \mathcal{B}$. The set of $G(\mathbb{K})$-orbits in $\mathcal{B} \times \mathcal{B}$ is known $[\mathrm{GV}]$ to be in (1-1)-correspondence with the set of $\mathbb{Z} \times \mathbb{Z}$-matrices $\left\|a_{i j}\right\|$ subject to the following conditions :

(i) the entries of the matrix are non-negative integers such that, for any $i, j \in \mathbb{Z}$, we have $a_{i+n, j+n}=a_{i, j}$;

(ii) the matrix $\left\|a_{i j}\right\|$ has finitely many non-vanishing diagonals, i.e., there exists $k>>0$ (depending on the matrix) such that $a_{i j}=0$ whenever $|i-j|>k$;

(iii) $\quad \Sigma_{1 \leq i \leq n} \Sigma_{j \in \mathbb{Z}} a_{i j}=d$.

The group $G(\mathbb{K})$ is an infinite-dimensional complex (algebraic) group. Similarly, the set $\mathcal{B}$ acquires the natural structure, cf. [KL 1], of an infinite dimensional complex variety, a direct limit of finite-dimensional projective varieties of increasing dimension. Next, we define $T^{*} \mathcal{B}$, a kind of "cotangent bundle" to $\mathcal{B}$ (which is not a vector bundle , cf. [Gi]), as follows. 
Call an endomorphism $x \in \mathfrak{g l}_{d}(\mathbb{K})$ regular semisimple (see [KL 3]) if it becomes regular semisimple after an extension of scalars from $\mathbb{K}$ to $\overline{\mathbb{K}}$, an algebraic closure of $\mathbb{K}$. Further, for any endomorphism $x \in \mathfrak{g l}_{d}(\mathbb{K})$ write its characteristic polynomial

$$
\operatorname{det}(\lambda \cdot I d-x)=\lambda^{d}+\ldots \in \mathbb{K}[\lambda]=\mathbb{C}((z))[\lambda] .
$$

Let $\mathcal{N}_{\text {rs }}$ be the set of all regular semisimple endomorphisms of $\mathbb{K}^{d}$ such that its characteristic polynomial is of the form $\operatorname{det}(\lambda \cdot I d-x)=\lambda^{n} \cdot P(\lambda, z)$ for some $P \in \mathbb{C}[[z]][\lambda]$ (no negative powers of $z$ !). It is clear that $0 \notin \mathcal{N}_{\text {rs }}$ and that, for any $x \in \mathcal{N}_{\text {rs }}$, there exists a lattice $L \subset \mathbb{K}^{d}$ such that $x(L) \subset L$ and, moreover, $x^{n}(L) \subset z \cdot L$. We define the cotangent bundle to $\mathcal{B}$ as follows

$$
T^{*} \mathcal{B}=\left\{(F, x) \in \mathcal{B} \times \mathcal{N}_{r s} \mid x\left(F_{i}\right) \subset F_{i-1}, \forall i \in \mathbb{Z}\right\}
$$

Dilation automorphisms $z \mapsto a \cdot z, a \in \mathbb{C}^{*}$ of the field $\mathbb{C}((z))$ give rise to a natural "rotation of the loop" $\mathbb{C}^{*}$-action on all the objects above.

Following the pattern of [KL 2], [Gi], and [GV], we introduce a subvariety $Z \subset T^{*}(\mathcal{B} \times \mathcal{B})$ to be the union of the conormal bundles to the $G(\mathbb{K})$-orbits in $\mathcal{B} \times \mathcal{B}$. Clearly the variety $Z$ is $G(\mathbb{K})$-stable with respect to the diagonal action. Thus, $Z$ may be viewed as a $\left(\mathbb{C}^{*} \ltimes G(\mathbb{K})\right) \times \mathbb{C}^{*}$ variety, where the first copy of $\mathbb{C}^{*}$ accounts for the "rotation of the loop" action, and the last copy accounts for dilations along the fibers of the cotangent bundle. Set $\mathbf{G}=\left(\mathbb{C}^{*} \ltimes G(\mathbb{K})\right) \times \mathbb{C}^{*}$, and let $K^{\mathbf{G}}(Z)$ denote the Grothendieck group of G-equivariant coherent (in an appropriate sense) sheaves on $Z$.

There is a convolution-type product on $K^{\mathbf{G}}(Z)$ defined as follows (cf. [GV], [CG]). Let $p_{i j}: T^{*} \mathcal{B} \times T^{*} \mathcal{B} \times T^{*} \mathcal{B} \rightarrow T^{*} \mathcal{B} \times T^{*} \mathcal{B}$ denote the projection along the factor not named. Each fiber of the map

$$
p_{13}: p_{12}^{-1}(Z) \cap p_{23}^{-1}(Z) \rightarrow T^{*} \mathcal{B} \times T^{*} \mathcal{B}
$$

was shown in [KL 3], to be a disjoint union of finite dimensional projective varieties. Moreover, the image of this map equals $Z$. Given G-equivariant sheaves $\mathcal{F}, \mathcal{F}^{\prime}$ on $Z$, define convolution $K^{\mathbf{G}}(Z) \otimes K^{\mathbf{G}}(Z) \rightarrow K^{\mathbf{G}}(Z)$ by the formula

$$
[\mathcal{F}] \star\left[\mathcal{F}^{\prime}\right]=\left(R p_{13}\right)_{*}\left(p_{12}^{*} \mathcal{F} \bigotimes_{\mathcal{O}_{T^{*} \mathcal{B} \times T^{*} \mathcal{B} \times T^{*} \mathcal{B}}}^{\mathbb{L}} p_{23}^{*} \mathcal{F}^{\prime}\right)
$$

We have the following toroidal analogue of [GV 1, theorem 7.9] : 
Theorem 5.1. There is a surjective algebra homomorphism

$$
\mathbf{U} \rightarrow K^{\left(\mathbb{C}^{*} \ltimes G(\mathbb{K})\right) \times \mathbb{C}^{*}}(Z) .
$$

All the homomorphisms above tend to become bijective as $d \rightarrow \infty$. The central extra-generator $D$ in $\mathbf{U}$ goes under the morphism of theorem 5.1 to the generator of the representation $\operatorname{ring} R\left(\mathbb{C}^{*}\right) \simeq \mathbb{Z}\left[D, D^{-1}\right]$, where the group $\mathbb{C}^{*}$ acts via the "rotation of the loop". Theorem 4.2 and Theorem 5.1 may be viewed as the two components of an analogue of the Satake isomorphism 1.1.

\section{Acknowledgements}

The first author is pleased to thank D. Kazhdan for a discussion a few years ago that has lead eventually to the $K$-theoretic construction of $\S 5$. We are especially grateful to H. Nakajima for providing us with a revised unpublished version of [Na2]. Theorems 2.4 and 4.2 above are based on the same idea as the results of [Na2] for ALE-spaces.

\section{References}

[BBD] A. Beilinson, J. Bernstein and P. Deligne, Faisceaux pervers, Astérisque 100 (1981).

[BLM] A. Beilinson, G. Lusztig and R. MacPherson, A geometric setting for quantum groups, Duke Math. J. 61 (1990), 655-675.

[Ch] I. Cherednik, Double Affine Hecke algebras, KZ-equations and Macdonald's operators, Intern. Math. Research Notices, Duke Math. J., 9 (1992), 171-180.

[CG] N. Chriss, V. Ginzburg, Representation theory and complex geometry (Geometric technique in representation theory of reductive groups), Progress in Mathem. Birkhäuser, 1995 (to appear).

[Dr 1] V. Drinfeld, Quantum Groups, Proceedings of the ICM, Berkeley 1986.

[Dr 2] _ A new realization of Yangians and quantum affine algebras, Soviet Math. Dokl. 36 (1988), 212-216.

[Dr 3] - Two-dimensional l-adic representations of the fundamental group of a curve over a finite field and automorphic forms on GL(2), Amer. J. Math. 105 (1983), 85-114.

[Gi] V. Ginzburg, Lagrangian construction of the universal enveloping algebra $U\left(s l_{n}\right)$, C. R. Acad. Sci. 312 (1991), 907-912.

[GKV] V. Ginzburg, M. Kapranov, and E. Vasserot, Elliptic algebras and equivariant elliptic cohomology, (to appear in q-alg eprints).

[GV] V. Ginzburg and E. Vasserot, Langlands reciprocity for affine quantum groups of type $A_{n}$, Intern. Math. Research Notices, Duke Math. J., 69, 1993, p. 67-85.

[IM] N. Iwahori and H. Matzumoto, On some Bruhat decompositions and the structure of the Hecke ring of a p-adic group, Publ. Math. I.H.E.S. 25 (1965), 5-48.

[Ji] M. Jimbo, A q-analogue of the enveloping algebra, Hecke algebra and the YangBaxter equation, Lett. in Math. Phys. 11 (1986), 247-252.

[KL 1] D. Kazhdan and G. Lusztig, Schubert varieties and Poincaré duality, Proc. Symp. Pure Math. 36 (1980). 
[KL 2] _ Proof of the Deligne-Langlands conjecture for affine Hecke algebras, Invent. Math. 87 (1987), 153-215.

[KL 3] _ The fixed point variety in affine flag manifold, Israel J. Math. 62 (1988), 129.

[K] K. Kodaira, On the structure of compact complex analytic surfaces, Amer. J. Math. 86 (1964), 751; 88 (1966), 682; 90 (1969), 55.

[Ko] S. Kondo, Enriques surfaces with finite automorphism groups, Japanese J. Math. 12 (1986), 191-282.

[L] R. Langlands, Problems in the theory of automorphic forms, Lect. Notes in Math. 170 (1970), 18-86.

[La] G. Laumon, Correspondence de Langlands géométrique pour les corps de functions, Duke Math. J. 54 (1987), 309-359.

[Lu] G. Lusztig, Quivers, perverse sheaves, and quantized enveloping algebras, Journ. A. M. S. 4 (1991), 365-421.

[Ma] M. Maruyama, Elementary transformations in the theory of algebraic vector bundles, in Algebraic Geometry, Lecture Notes in Math. 961, Springer-Verlag, Berlin, 1983, p. 241-266.

[MRY] R. V. Moody, S. E. Rao, and T. Yokonuma, Toroidal Lie algebras and vertex representations, Geometricae Dedicata 35 (1990), 283-307.

[Na 1] H. Nakajima, Instantons on ALE-spaces, quiver varieties, and Kac-Moody algebras, Duke Math. J. 76, 2, (1994).

[Na 2] _ Gauge theory on resolutions of simple singularities and simple Lie algebras, Intern. Mathem. Research Notices. 2 (1994), 61-74.

[Né] A. Néron, Modèles minimaux des variétés Abéliennes sur les corps locaux et globaux, Publ. Mathém. IHES. 21 (1964), 5-126.

[Ta] J. T. Tate, Algorithm for determining the type of singular fiber in an elliptic pencil, Lecture Notes in Math. 476 (1975), 33-52.

[VW] C. Vafa and E. Witten, A strong coupling test of S-duality, preprint, 1994.

[Wi] E. Witten, Topological quantum field theories, Commun. Math. Phys. 117 (1988), 353.

Department of Mathematics, University of Chicago, Chicago, IL 60637

E-mail address: ginzburg@math.uchicago.edu

Department of Mathematics, Northwestern University, Evanston, IL 60208

E-mail address: kapranov@chow.math.nwu.edu

Ecole Normale Supérieure, 45 Rue D'Ulm, 75005 Paris, FRANCE

E-mail address: vasserot@dmi.ens.fr 